Akkerman, A.E., Kuyvenhoven, M.M., Verheij, T.J.M., Dijk, L. van. Antibiotic use in Dutch general practice: nationwide electronic GP database and national reimbursement rates.

Pharmacoepidemiology and Drug Safety: 2008, 17(4), 378-383

\begin{tabular}{|l|l|}
\hline Postprint Version & 1.0 \\
\hline Journal website & $\underline{\text { http://www3.interscience.wiley.com/journal/116329891/abstract }}$ \\
\hline $\begin{array}{l}\text { Pubmed link } \\
\text { http://www.ncbi.nlm.nih.gov/sites/entrez?Db=pubmed\&Cmd=ShowDetailView } \\
\text { \&TermToSearch=17929333 }\end{array}$ \\
\hline DOI & $\underline{10.1002 / \text { pds.1501 }}$ \\
\hline
\end{tabular}

This is a NIVEL certified Post Print, more info at http://www.nivel.eu

\title{
Antibiotics in Dutch general practice: nationwide electronic GP database and national reimbursement rates
}

\author{
ANNEMIEK E. AKKeRMAN PHD ${ }^{1 *}$, MARIJKE M. KUYVENHOVEN PHD ${ }^{1}$, THEO J.M. VERHEIJ \\ PHD $^{1}$ AND LISET VAN DIJK PHD ${ }^{2}$ \\ ${ }^{1}$ Julius Center for Health Sciences and Primary Care, University Medical Center (UMC) \\ Utrecht, Utrecht, The Netherlands \\ ${ }^{2}$ Netherlands Institute for Health Services Research (NIVEL), Utrecht, The Netherlands
}

\section{SUMMARY}

Purpose In order to assess whether different databases generate information which can be reliable compared with each other, this study aimed to assess to which degree prescribing rates for systemic antibiotics from a nationwide electronic general practitioner (GP) database correspond with national reimbursement rates, and to investigate for which indications antibiotics are prescribed.

Methods Nationwide GP prescribing data were collected from the Second Dutch National Survey of General Practice (DNSGP-2) based on 90 general practices serving 358008 patients in 2001. Dutch national reimbursement rates for GPs were derived from claims data of the Dutch Drug Information System/Health Care Insurance Board (GIP/CVZ) from 2001. We calculated antibiotic prescribing rates per 1000 patients/inhabitants for each database, and these rates were compared for the total rates and according to antibiotic subgroups. Indications for which GPs prescribed antibiotics were described.

Results In national reimbursement data, 339 antibiotic prescriptions per 1000 inhabitants were prescribed by GPs, while the nationwide GP database showed 255 prescriptions per 1000 patients (75\% coverage with reimbursement rates). The nationwide GP database showed high volumes of sulphonamides \& trimethoprim, and small volumes of macrolides and quinolones. Half of the prescriptions (48\%) were prescribed for respiratory diseases, a quarter (26\%) for urinary diseases and 7\% for ear diseases.

Conclusions GPs voluntarily participating in a research network prescribe less antibiotics than Dutch GPs in general, and are cautious in prescribing newer and more broad-spectrum antibiotics. This point has to be taken into account when databases will be compared with each other. 
Akkerman, A.E., Kuyvenhoven, M.M., Verheij, T.J.M., Dijk, L. van. Antibiotic use in Dutch general practice: nationwide electronic GP database and national reimbursement rates. Pharmacoepidemiology and Drug Safety: 2008, 17(4), 378-383

\section{INTRODUCTION}

Internationally, research in antimicrobial use is growing, while a variety of health care databases are used. However, these databases substantially differ with regard to their quality and completeness. 1 Next to this, volumes are calculated as number of prescriptions as well as daily defined dosages (DDDs). So, it is important to assess whether these different databases generate information which can be reliable compared with each other. Moreover, it is always a major issue to value databases used in research.

In the Netherlands, antibiotic use by general practitioners (GPs) can be assessed with the aid of the national rates from the Health Insurance Companies (Dutch Drug Information System/Health Care Insurance Board; GIP/CVZ).2 However, studies about antimicrobial use in general practice need the opportunity to give insight into the indications for which GPs prescribe antibiotics, and reimbursement rates lack these indications. Therefore, antibiotic prescribing data derived from electronic GP databases are frequently used in such studies.35 A disadvantage of electronic GP databases is that they yield prescriptions as rates, and lack information to calculate DDDs. In addition, a small study among 39 GPs has shown that GPs intensively using electronic medical record systems seem to have about $20 \%$ lower antibiotic prescribing rates than the remaining GPs in their region.6 In order to get better insight into the correspondence between the antibiotic prescription rates of electronic GP databases and national reimbursement rates, this study aimed to assess to what extent a nationwide GP database of 186 GPs from the Second Dutch National Survey of General Practice (DNSGP2), corresponded with national reimbursement prescribing rates of Dutch GPs, concerning all patients in 2001. Next to that, we investigated antibiotic prescribing rates according to antibiotic subgroups and antibiotic indications, to get a more detailed insight into the use of antibiotics in Dutch general practice.

\section{METHODS}

\section{Design}

A cross-sectional study, comparing antibiotic prescribing rates from the nationwide GP database DNSGP-2 with national GP reimbursement rates from GIP/CVZ in 2001.

\section{Data sources}

\section{Nationwide GP database.}

Antibiotic prescribing rates from the nationwide GP database DNSGP-2, carried out by the Netherlands Institute for Health Services Research (NIVEL), were used.7 The research network of this project, performed in 2001, included 195 GPs in 104 practices (volunteers). For our analyses, we used a previously described selection of 186 GPs in 90 practices serving 358008 patients (mid-year population) (reasons for exclusion: inadequate registration and software problems).8 The GPs were considered representative for all Dutch GPs (N=7217). No statistically significant differences for age, gender, region of residence and urbanisation were found, except for type of practice: single handed practices were underrepresented.9 The patients captured by the practices are a good representation of the Dutch population on age, gender and type of health insurance.7 GPs registered data about all patient contacts during 12 months. Prescriptions were registered in a separate database and were coded according to the WHO Anatomical Therapeutic Chemical (ATC) classification10 with additional information about indication (in International Classification of Primary Care (ICPC) format), dates and patient identification. Next to above-mentioned selection, practices who recorded ICPC codes (antibiotic indications) in less than $60 \%$ of all antibiotic prescriptions were excluded also (three practices).

The mid-year population became 340565 patients. 
Akkerman, A.E., Kuyvenhoven, M.M., Verheij, T.J.M., Dijk, L. van. Antibiotic use in Dutch general practice: nationwide electronic GP database and national reimbursement rates. Pharmacoepidemiology and Drug Safety: 2008, 17(4), 378-383

\section{National reimbursement data.}

National GP reimbursement rates were derived from data of the Dutch Drug Information System/Health Care Insurance Board (GIP/CVZ). GIP/CVZ collects prescription data at the level of provider (e.g. GP, medical specialist and dentist) and patient (age, gender, region). Prescribing rates are based on prescription utilisation databases, referring to a representative sample of 6.8 million compulsory insured patients in 200111 from regions all over the Netherlands and, standardised to the agegender distribution, extrapolated to the total population of Dutch inhabitants ( $\mathrm{N}=16105285$ inhabitants).

National antibiotic prescribing rates of GPs were estimated by calculating the mean number of antibiotic prescriptions in 2001 attributable to GP prescribing per 1000 inhabitants per year. Drugs were coded according to ATC classification and calculations were carried out by GIP/CVZ.

\section{Subgroups of antibiotics}

This study described prescriptions of antibiotics for systemic use (ATC code J01) with the following subgroups: (a) tetracyclines (J01 AA), (b) narrowspectrum penicillin (J01 CE/CF), (c) broad-spectrum penicillin (J01 CA-amoxicillin/J01 CR-amoxicillin- clavulanate), (d) sulphonamides \& trimethoprim (J01 E), (e) macrolides (J01 F), (f) quinolones (J01 MA), (g) nitrofurantoin (J01 XE) and (h) others (J01 D, G, XA-XD, XX).

\section{Outcome measures}

Outcome measures were (a) antibiotic prescribing rates per 1000 patients/inhabitants per year prescribed by GPs in 2001 and (b) antibiotic prescribing rates according to antibiotic subgroups (per ATC chapter) and antibiotic indications (per ICPC chapter).

\section{Analysis}

The antibiotic prescribing rates per 1000 patients/ inhabitants from both sources were compared for the total rate, and according to antibiotic subgroups, by calculating the coverage percentage (correspondence of the nationwide GP database rates with national reimbursement rates; calculation: nationwide GP database rate divided by national reimbursement rate). After that, antibiotic prescribing rates according to antibiotic subgroups and antibiotic indications were described, using rates from the GP database DNSGP-2. Data were analysed with SSPS version 12.0 (frequencies).

\section{[TABLE 1]}

\section{RESULTS}

\section{Antibiotic prescribing rates}

National reimbursement data showed that 405 antibiotic prescriptions per 1000 inhabitants were out-patiently prescribed in the Netherlands in 2001, of which $86 \%$ were prescribed by GPs, being 339 antibiotic prescriptions per 1000 inhabitants. The nationwide GP database DNSGP-2 showed a lower prescription volume: 255 antibiotic prescriptions per 1000 patients for systemic antibiotics, which covers $75 \%$ of the national reimbursement rate (Table 1). This means that GPs participating in the research network of the nationwide GP database DNSGP-2 prescribe 25\% less antibiotics than Dutch GPs in general. The coverage percentages (correspondence of nationwide GP database rates with national reimbursement rates) showed lower volumes in all subgroups, but relatively high volumes of sulphonamides \& trimethoprim (97\%) and relatively small volumes of macrolides and quinolones (60\%) (Table 1). This indicates that GPs voluntarily participating in the research network of the nationwide GP database DNSGP-2 prescribe as much sulphonamides \& trimethoprim as Dutch GPs in general, but are more cautious in prescribing macrolides and quinolones. 
Akkerman, A.E., Kuyvenhoven, M.M., Verheij, T.J.M., Dijk, L. van. Antibiotic use in Dutch general practice: nationwide electronic GP database and national reimbursement rates. Pharmacoepidemiology and Drug Safety: 2008, 17(4), 378-383

\section{[TABLE 2]}

\section{Antibiotic prescriptions per ICPC chapter}

The health problems, for which antibiotics were prescribed, were distributed among ICPC chapters. In 87\% of all antibiotic prescriptions indications (in ICPC format) were recorded.

Half of all antibiotic prescriptions (48\%) were indicated for respiratory diseases (ICPC chapter R) and a quarter (26\%) for urinary diseases (ICPC chapter U), while skin diseases (S) and ear diseases $(\mathrm{H})$ covered, respectively, 10 and $7 \%$ of all antibiotic prescriptions. The remaining $9 \%$ antibiotic prescriptions were indicated for the remaining ICPC chapters, of which $2 \%$ for digestive diseases (ICPC chapter D).

Looking more specific at the subgroups of antibiotics in Table 2, it can be seen that around $80 \%$ of tetracylines, amoxicillin and macrolides, as well as half $(45+8=53 \%)$ of amoxicillinclavulanate, were prescribed for respiratory and ear diseases. Almost half (47\%) of all narrow-spectrum penicillins were prescribed for respiratory diseases and $43 \%$ for skin diseases. Sulphonamides \& trimethoprim, quinolones and nitrofurantion were mostly prescribed for urinary diseases. The other subgroups of antibiotics were about equally prescribed for all ICPC categories.

\section{DISCUSSION}

Although there is a clear correlation between prescribing rates for systemic antibiotics from a nationwide electronic GP database and national reimbursement rates, there are some relevant differences.

GPs voluntarily participating in a nationswide research network, using electronic medical record systems, prescribe a quarter (25\%) less antibiotics compared with national reimbursement rates from the Health Insurance Companies, with relatively high volumes of sulphonamides \& trimethoprim and relatively small volumes of macrolides (mainly for respiratory tract infections) and quinolones (mainly for urinary tract infections). About 5 of each 10 antibiotic prescriptions are indicated for respiratory tract infections, a quarter for urinary diseases and 7\% for ear diseases (mostly acute otitis media).

\section{Strengths and limitations of the study}

\section{KEY POINTS}

- Antibiotic prescribing rates from a nationwide electronic general practice database correspond with national reimbursement rates.

- However, general practitioners voluntarily participating in a research network are more cautious in prescribing antibiotics and especially prescribe less macrolides and quinolones than the total population of general practitioners.

- About half of antibiotic prescriptions in general practice are prescribed for respiratory tract diseases, a quarter for urinary tract diseases and $7 \%$ for ear diseases.

Characteristics of GPs and practices from the nationwide GP database DNSGP-2 did not differ from those of the total population of Dutch practices, except for type of practice: single handed GPs were underrepresented in the study population. So, in general, the nationwide GP database DNSGP-2 is believed to give a representative impression of morbidity and prescribing habits in Dutch General Practice.7 Although there was no absolute guarantee that medication prescribed in evening, night and weekend shift or during home visits are completed in the medical records, the difference of $25 \%$ less prescriptions 
Akkerman, A.E., Kuyvenhoven, M.M., Verheij, T.J.M., Dijk, L. van. Antibiotic use in Dutch general practice: nationwide electronic GP database and national reimbursement rates. Pharmacoepidemiology and Drug Safety: 2008, 17(4), 378-383

compared with the national reimbursement data cannot be explained by a possible lack of these prescriptions. The vast majority ofmedical information about out-of-hours care is transferred to GPs' offices. Next to this, in order to participate in the research network, GPs were required to have fully computerised medical files to ensure completeness of data. Therefore, it is reasonable to assume that almost all data with regard to antibiotic prescriptions were available.

Most GPs participating in the research network of the GP database DNSGP-2 did use ICPC codes for antibiotic indications in more than $85 \%$ of all prescriptions.

Inclusion of three GP practices who recorded ICPC codes (antibiotic indications) in less than $60 \%$ of all antibiotic prescriptions afterwards, did not alter the results.

The national reimbursement data of GIP/CVZ concerned 6.8 million compulsory insured patients, being $67 \%$ of all Dutch compulsory insured patients, and extrapolated the rates for the total population (compulsory as well as privately insured patients). The rates are assumed to give a reliable indication of the total amount of prescriptions in Dutch General Practice. ${ }^{11}$ The data in the study are from 2001. There is no reason to assume that antibiotic prescription rates, and especially the differences between databases, have substantially changed since then. As we pointed out in another publication, the low Dutch prescription rates slowly change over time. ${ }^{2}$ Since 2001, DDDs per 1000 inhabitants have shown a small decrease for sulphonamides \& trimethoprim and a small increase for macrolides and nitrofurantoin. ${ }^{12}$

\section{Comparison with existing literature}

The results of our study corroborate the findings of an older and smaller study which showed that GPs using electronic medical record systems seem to have lower antibiotic prescribing rates than the remaining GPs in their region. ${ }^{6}$ In line with this previous study, our findings indicate that GPs participating in the research network of the nationwide GP database DNSGP-2, being volunteers, more strongly adhere to guidelines than nonparticipating GPs. This difference appears to result in lower antibiotic prescribing rates and relatively small volumes of macrolides and quinolones.

The finding that practices participating in a research network of nationwide GP database prescribe about a quarter fewer antibiotics than GPs in general, is supported by a study in England. ${ }^{13,14}$ The distribution of antibiotics among ICPC chapters is nearly the same as in the UK, except for urinary diseases; in the Netherlands $25 \%$ of all antibiotics, in the UK $10 \% .{ }^{15}$ Half of all antibiotic prescriptions were indicated for respiratory and ear diseases, while 20 years ago $70 \%$ of antibiotic prescriptions were attributed to these disease categories in the Netherlands. ${ }^{16}$ Such a reduction has also been shown by Otters et al., concerning paediatric antibiotic prescribed in the Netherlands. ${ }^{5}$ Reducing antibiotic prescribing rates for respiratory tract infections have also been described for the UK, probably being mostly caused by a changing inclination of patients to consult their GP for respiratory symptoms rather than by a changing GP threshold to prescribe antibiotics in the UK as well as in the Netherlands. ${ }^{7,8,13,17}$ Most of tetracyclines, amoxicillin and macrolides were prescribed for respiratory and ear tract diseases, while most of sulphonamides \& trimethoprim, quinolones and nitrofurantoin were prescribed for urinary tract diseases. In main lines, this distribution is the same in the $\mathrm{UK}^{15}$

\section{CONCLUSIONS}

Antibiotic prescribing rates from a nationwide electronic GP database correspond, in main lines, with nationwide reimbursement rates. However, GPs participating in research networks as DNSGP-2, prescribe fewer antibiotics than the total population of GPs, and are cautious in prescribing newer and more broad-spectrum antibiotics. When using data from GP research networks for scientific purposes, this should be taken into account. 
Akkerman, A.E., Kuyvenhoven, M.M., Verheij, T.J.M., Dijk, L. van. Antibiotic use in Dutch general practice: nationwide electronic GP database and national reimbursement rates. Pharmacoepidemiology and Drug Safety: 2008, 17(4), 378-383

\section{ACKNOWLEDGEMENTS}

We gratefully thank Ms C. Hooijkaas and Mr J.F.Piepenbrink of the Dutch Drug Information System/ Health Care Insurance Board (GIP/CVZ) for the national reimbursement rates attributable to GP prescribing.

We are very grateful to all general practitioners and patients who voluntarily participated in the Second Dutch National Survey of General Practice, and Peter Zuithoff for his datamanagement activities.

\section{REFERENCES}

1. Black N, Barker M, Payne M. Cross sectional survey of multicentre clinical databases in the United Kingdom. Br Med J 2004; 328: 1478-1482.

2. Kuyvenhoven MM, van Balen FAM, Verheij TJM. Outpatient antibiotic prescriptions from 1992 to 2001 in the Netherlands.

J Antimicrob Chemother 2003; 52: 675-678. DOI: 10.1093/jac/ dkg412

3. Welschen I, Kuyvenhoven MM, Hoes AW, Verheij TJM. Effectiveness of a multiple intervention to reduce antibiotic prescribing for respiratory tract symptoms in primary care: randomised controlled trial. Br Med J 2004; 329: 431-435. DOI: 10.1136/ bmj.38182.591238.EB

4. Akkerman AE, van der Wouden JC, Kuyvenhoven MM, Dieleman JP, Verheij TJM. Antibiotic prescribing for respiratory tract infections in Dutch primary care in relation to patient age and clinical entities. J Antimicrob Chemother 2004; 54: 1116-1121.

DOI: 10.1093/jac/dkh480

5. Otters HBM, van der Wouden JC, Schellevis FG, van Suijlekom- Smit LWA, Koes BW. Trends in prescribing antibiotics for children in Dutch general practice. J Antimicrob Chemother 2004; 53: 361-366. DOI: 10.1093/jac/dkh062

6. Westert GP, Hoonhout LHF, de Bakker DH, van den Hoogen HJM, Schellevis FG. Huisartsen met en zonder elektronisch medisch dossier: weinig verschil in medisch handelen. [General practitioners with and without electronic patient records: little variation in medical care]. Huisarts Wet 2002; 45: 58-62.

7. Westert GP, Schellevis FG, de Bakker DH, Groenewegen PP, Bensing JM, van der Zee J. Monitoring health inequalities through general practice: the Second Dutch National Survey of General Practice. Eur J Public Health 2005; 15: 59-62. DOI: 10.1093/eurpub/ckil16 8. Kuyvenhoven MM, van Essen GA, Schellevis FG, Verheij TJM.

Management of upper respiratory tract infections in Dutch general practice; antibiotic prescribing rates and incidences in 1987 and 2001. Fam Pract 2006; 23: 175-179. DOI: 10.1093/fampra/cmil22

9. Florentinus SR, Heerdink ER, de Boer A, van Dijk L, Leufkens HGM. The trade-off between cardiovascular and gastrointestinal effects of rofecoxib. Pharmacoepidemiol Drug Saf 2005; 14: 437-441. DOI:

10.1002/pds.1123 10. WHO Collaborating Centre for Drugs Statistics Methodology. http://www.whocc.no/atcddd/ [accessed: August 27, 2007].

11. The Dutch Drug Information System of the Health Care Insurance Board. http://www.gipdatabank.nl/ [accessed: August 27, 2007].

12. SWAB. NethMap 2007-Consumption of antimicrobial agents and antimicrobial resistance among medically important bacteria in The Netherlands http://www.swab.nl $>$ professional or foreign visitor>NethMap 2007, [accessed: August 27, 2007].

13. Ashworth M, Latinovic R, Charlton J, Cox K, Rowlands G, Gulliford M. Why has antibiotic prescribing for respiratory illness declined in primary care? A longitudinal study using the General Practice Research Database. J Public Health (Oxf) 2004; 26: 268-274. DOI: 10.1093/pubmed/fdh160

14. Fleming DM, Ross AM, Cross KW, Kendall H, Elliot AJ. Concerning: 'Why has antibiotic prescribing for respiratory illness declined in primary care? A longitudinal study using the General Practice Research Database'. J Public Health (Oxf) 2005; 27: 228-229.

15. Petersen I, Hayward AC. Antibacterial prescribing in primary care. J Antimicrob Chemother 2007; 60 (Suppl. 1): i43-i47.DOI: 10.1093/jac/dkm156 
Akkerman, A.E., Kuyvenhoven, M.M., Verheij, T.J.M., Dijk, L. van. Antibiotic use in Dutch general practice: nationwide electronic GP database and national reimbursement rates. Pharmacoepidemiology and Drug Safety: 2008, 17(4), 378-383

16. Sampers GHMA, Sturm AW. Antimicribie"le middelen in de eerste lijn: onderzoek bij alle huisartsen in een middelgrote stad [Antimicrobial agents in primary health care: study of all family physicians in a medium-sized city]. Ned Tijdschr Geneeskd 1998; 132: 676-680.

17. Fleming DM, Ross AM, Cross KW, Kendall $\mathrm{H}$. The reducing incidence of respiratory tract infection and its relation to antibiotic prescribing. $\mathrm{Br} J$ Gen Pract 2003; 53: 778-783. 
Akkerman, A.E., Kuyvenhoven, M.M., Verheij, T.J.M., Dijk, L. van. Antibiotic use in Dutch general practice: nationwide electronic GP database and national reimbursement rates.

Pharmacoepidemiology and Drug Safety: 2008, 17(4), 378-383

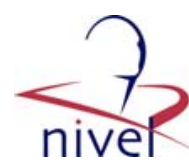

\section{TABLES}
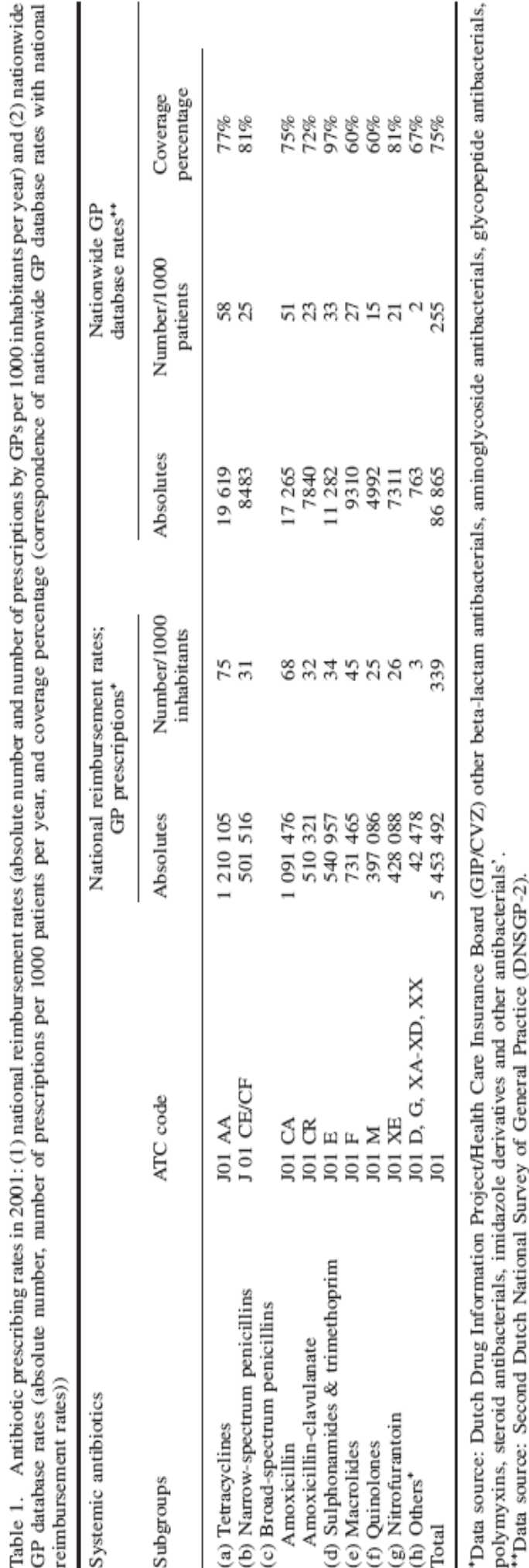

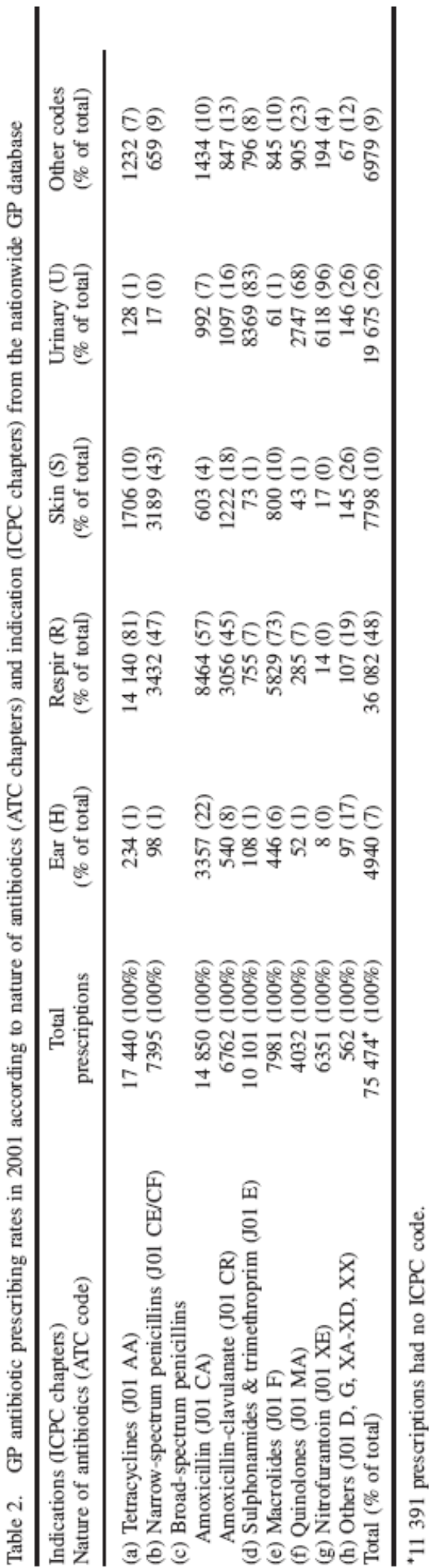

